\title{
Cause and Effect Relationship between Changes in Scleral Matrix Metallopeptidase-2 Expression and Myopia Development in Mice
}

Fei Zhao, ${ }^{{ }^{\dagger}}$ Qingyi Zhou, ${ }^{{ }^{\dagger}}$ Peter S. Reinach, ${ }^{* \dagger}$ Jinglei Yang, ${ }^{{ }^{\dagger}}$ Li Ma, ${ }^{{ }^{*}}$ Xiaojun Wang, ${ }^{{ }^{*}}$ Yingying Wen, ${ }^{{ }^{\dagger}}$ Nethrajeith Srinivasalu, ${ }^{* \dagger}$ Jia Qu, ${ }^{* \dagger}$ and Xiangtian $\mathrm{Zhou}^{* \dagger}$

From the School of Optometry and Ophthalmology and Eye Hospital, * Wenzhou Medical University, Wenzhou, Zhejiang; and the State Key Laboratory of Optometry, Ophthalmology, and Vision Science, ${ }^{\dagger}$ P. R. China and Zhejiang Provincial Key Laboratory of Ophthalmology and Optometry, Wenzhou, Zhejiang, People's Republic of China

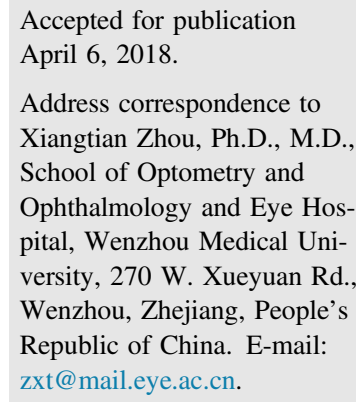

\begin{abstract}
Myopia is a serious sight-compromising condition in which decreases in scleral biomechanical strength are associated with protease up-regulation resulting in thinning of its collagenous framework and changes in the extracellular matrix composition. Matrix metallopeptidase (MMP)-2 is one of the known proteases mediating these alterations. To determine whether MMP-2 up-regulation precedes myopia development, the direct effects of gain and loss in Mmp2 gene function were evaluated on refractive development and form deprivation myopia in mice. Four weeks after injecting an adeno-associated virus serotype 8 packaged Mmp2 overexpression vector (AAV8-Mmp2), scleral MMP-2 up-regulation was accompanied by significant myopia in a normal visual environment. In contrast, AAV8 packaging with shRNA targeting Mmp2 inhibited rises in MMP-2 expression induced by form deprivation by $54 \%$ and reduced myopia development by $23 \%$ compared with eyes injected with an irrelevant scrambled sequence. Because opposing changes in MMP-2 protein expression levels had corresponding effects on myopia progression, up-regulation of this protease contributes to inducing this condition. This notion of a cause-and-effect relationship between MMP-2 up-regulation and myopia development is supported by showing that form-deprived myopia development was attenuated by $27 \%$ in fibroblast-specific Mmp2 deletion ( $\mathrm{S} 100 a 4^{\mathrm{cre}} \mathrm{Mmp} 2^{f l / f l}$ ) mice relative to Cre-negative littermates $\left(\mathrm{Mmp} 2^{f l / f l}\right)$. Therefore, MMP-2 is a potential drug target for inhibiting myopia progression. (Am J Pathol 2018, 188: 1754-1767; https:// doi.org/10.1016/j.ajpath.2018.04.011)
\end{abstract}

Myopia is the most prevalent refractive error resulting in blurred vision, which in more severe cases can lead to retinal detachment, choroidal neovascularization, and possibly blindness. ${ }^{1}$ Despite the availability of low-dose atropine and orthokeratology to slow myopia progression, which can be implemented to treat high-risk myopia in children, atropine has limited effectiveness to slow myopia progression. Therefore, there is an urgent need to identify alternative strategies to reverse the structural changes in the posterior sclera that occur during myopia development. ${ }^{2}$ Myopia mostly develops as a consequence of excessive axial length (AL) elongation and increases in vitreous chamber depth (VCD). The changes are accompanied by declines in scleral biomechanical strength caused by collagen fiber thinning and extracellular matrix (ECM) remodeling identified in human sclera and other experimental models, ${ }^{3-7}$ The decline in type I collagen accumulation in large part accounts for most of the increases in

Supported by the National Natural Science Foundation of China (National Science Foundation of China) grants 81700868 (F.Z.), 81422007 (X.Z.), 81470659 (J.Q.), 81170870 (X.Z.), and 81670886 (X.Z.); the Scientific Bureau of Wenzhou City grant 2016Y0728 (F.Z.); the Natural Science Foundation of Zhejiang Province (Zhejiang Provincial Natural Science Foundation) grants LQ16H120006 (F.Z.) and LZ14H120001 (X.Z.); The Zhejiang Provincial Program for the Cultivation of High-Level Innovative Health Talents (X.Z.); and The National Young Excellent Talents Support Program (X.Z.).

F.Z. and Q.Z. contributed equally to this work.

Disclosures: None declared. 
scleral distensibility because its dry weight is equal to approximately $90 \%$ of the scleral dry weight. ${ }^{8,9}$ These losses result from synthesis inhibition along with increases in collagen degradation. ${ }^{10-18}$ Furthermore, ECM remodeling stems from cytokine up-regulation that mediates changes in scleral cell phenotype and up-regulation of a host of different types of proteases. ${ }^{19}$ However, it is unclear whether these increases in protease expression precede myopia development progression or develop as a consequence of myopia.

Matrix metallopeptidase (MMP)-2 up-regulation contributes to scleral framework restructuring and ECM remodeling in tree shrews, ${ }^{20,21}$ guinea pigs, $^{22}$ and chicks $^{19,23-25}$ during myopia, whereas during reversal of this process declines occur in scleral MMP-2 expression. In addition to promoting collagen degradation, MMP-2 upregulation contributes to mediating collagen remodeling by inhibiting collagen synthesis during fibrosis and tumor formation in other tissues. ${ }^{26-29}$ Furthermore, increases in the MMP-2 human aqueous levels are strongly correlated with AL elongation. ${ }^{30,31}$

Another factor that affects the ECM remodeling process is the balance between the levels of MMPs and their natural endogenous inhibitors, that is, tissue inhibitors of matrix metalloproteinases. ${ }^{21,22,24,32}$ The role of changes in tissue inhibitors of matrix metalloproteinase-2 expression in ECM remodeling is species specific because this inhibitor suppressed the activities of scleral MMPs and collagen degradation as well as myopia development in tree shrews, ${ }^{33}$ whereas it failed to inhibit myopia development in chicks. ${ }^{34}$ Even though MMP-2 involvement in myopia has been extensively characterized, heretofore genetically modified animals have not been used to determine whether there is a cause-and-effect relationship between MMP-2 upregulation and myopia in mice. To address this question, we contrasted the individual effects of either gain or loss of Mmp2 gene function on refractive development and myopia progression.

Here, the time-dependent effects of alterations in MMP-2 expression on eye growth in a normal vision environment were compared with those occurring in a vision-obstructed environment to induce myopia. To induce myopia, a welldescribed mouse myopia model was used in which visual acuity was disrupted by outfitting mice with translucent diffusers that degrade visual acuity. With this form deprivation (FD) model, parallel changes in type I collagen expression levels were used as an index for evaluating variations in scleral biomechanical strength that are associated with loss and gain of MMP-2 function. Furthermore, the role of MMP-2 up-regulation in initiating myopia was determined by comparing FD myopia (FDM) development in a fibroblast-specific Mmp2 deletion mouse with that in a Mmp 2 gene-modified mouse. The results show that FD induces scleral MMP-2 up-regulation which confirms that increases in MMP-2 expression contribute to initiating myopia development in mice.

\section{Materials and Methods}

\section{Animals}

Animal work in this study was approved by the Animal Care and Ethics Committee at Wenzhou Medical College, Wenzhou, China. All experiments were conducted in adherence with the Association for Research in Vision and Ophthalmology Statement for the Use of Animals in Ophthalmic and Visual Research. All of the mice were bred in the animal breeding unit at Wenzhou Medical College (Wenzhou, China). They were raised with a 12-hour light/12-hour dark cycle, and the luminance was approximately 100 to 200 lux.

\section{Generation of Fibroblast-Specific Mmp2-Deficient Mice}

Because this mutant was not available, the $M m p 2^{f / f t}$ mice were first developed on a C57BL/6J background in the Model Animal Research Center at Nanjing University (Nanjing, China). Two loxP sites were inserted flanking exon 2 of the Mmp2 gene and bred to mice expressing the Cre recombinase enzyme, which resulted in offspring with exon 2 deleted in the Cre-expressing tissue. Frame-shift caused by lack of exon 2 deletes an amino acid sequence causing MMP-2 dysfunction. ${ }^{35} \mathrm{Mmp} 2^{f / f l}$ mice were crossed with S100a4-Cre mice (stock number 012641; The Jackson Laboratory, Bar Harbor, ME) to generate fibroblast-specific Mmp2 deletion $\left(S 100 a 4^{\text {cre }} \mathrm{Mmp} 2^{f l f l}\right)$ mice, and the Cre negative littermate $\left(\mathrm{Mmp}^{f / / f}\right)$ mice served as the control. Figure 1, A-E, illustrates the detailed strategy used for obtaining the indicated targeted Mmp2 gene fibroblast deletion. PCR analysis of tail-extracted genomic DNA confirmed the predicted identity of the mice.

To determine whether there is Cre recombinase expression in S100a4-expressing scleral cells, the S100a4 ${ }^{\text {cre }}$ mice were crossed with the $R O S A^{m T / m G}$ mice (stock number 007676; The Jackson Laboratory) to generate $S 100 a 4^{c r e} R O S A^{m T / m G}$ mice and the Cre-negative littermate $\left(R O S A^{m T / m G}\right)$ mice. Cell membrane-localized tdTomato fluorescence (red) expression is widespread in cells in the $R O S A^{m T / m G}$ mice. Cre recombinase-expressing cells are evident where the red fluorescence was replaced by cell membrane-localized enhanced green fluorescent protein (GFP; green) expression in the $S 100 a 4^{\text {cre }} R_{O S A^{m T / m G}}$ mice.

\section{Induction of FDM and Ocular Biometric Measurements in Mice}

Monocular FD was induced by occluding the right eyes with translucent diffusers ${ }^{36}$ (Supplemental Figure S1). The formdeprived eyes are designated as FD-T, whereas the untreated left fellow eyes were designated as FD-F. Ocular refraction was measured in a dark room by using an eccentric infrared photorefractor. The ocular biometric variables, AL, VCD, corneal radius curvature (CRC), and lens thickness (LT), were measured by using a custom-made optical coherence tomograph. ${ }^{36}$ 

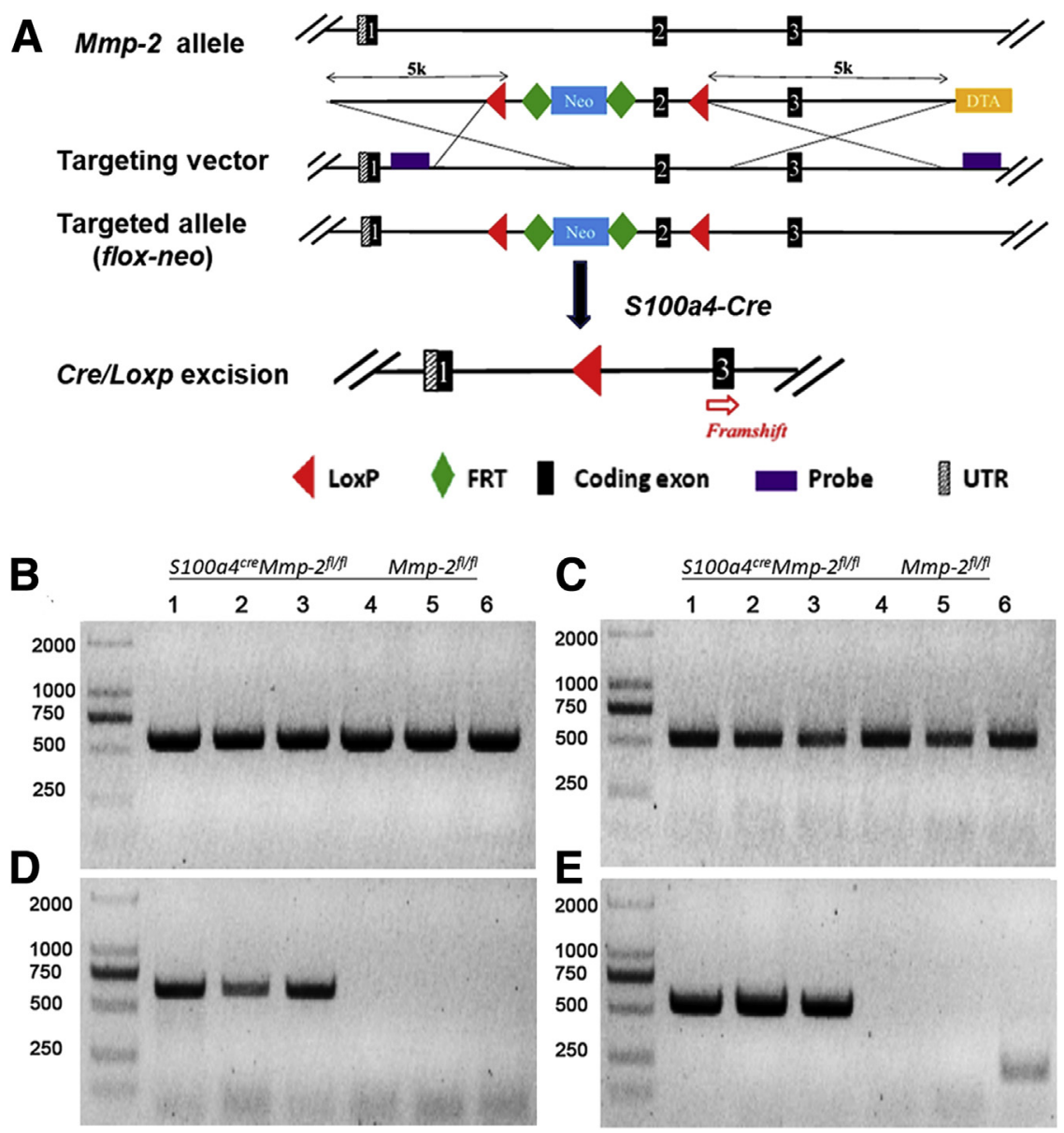

Figure 1 Strategy for targeted fibroblast Mmp2 gene deletion. A: Targeting of mice Mmp2 locus. LoxP-Neo cassette was inserted into the region between exons 1 and 2, and a single lox $P$ site was inserted in the region between exons 2 and 3 . After crossing with S100a4-Cre mice, exon 2 of Mmp2 was deleted. Agarose gel electrophoresis was performed to verify the PCR products amplified from genomic DNA of $S 100 a 4^{c r e} M m p 2^{f l f l}$ (lane 1 to 3 ) and $M m p 2^{f l / f l}$ (lane 4 to 6 ) mice. B-D: PCR identification of $5^{\prime}(\mathbf{B})$ and $3^{\prime}(\mathbf{C})$ loxP sites and $C r e$ recombinase sequence (D) predicted 568-, 524-, and 490-bp products, respectively, using the primers listed in Table 1. E: The primer pair for null listed in Table 1 amplified a fragment of approximately $615 \mathrm{bp}$ in $\$ 100 \mathrm{a}^{\mathrm{cre}} \mathrm{Mmp} 2^{\mathrm{fl} / \mathrm{fl}}$ mice but failed to generate any products in the $M m p 2^{f l f l}$ mice. FRT, flippase recognition target; UTR, untranslated region.

\section{Construction of AAV8-Mmp2 Overexpression Vector}

At Biowit Technologies (Shenzhen, China), the cDNA sequence (1989 bp) of mouse Mmp2 (NM_008610.3) was cloned into the adeno-associated virus serotype 8 (AAV8) plasmid that yielded AAV8-Mmp2. After sequence verification, amplicons were packaged by using a three-plasmid transient transfection of human embryonic kidney 293T cells (ATCC, Manassas, VA). The AAV8 supernatant was collected 48 hours after transfection, and the virus was released from the human embryonic kidney 293T cells through three consecutive freeze-thaw cycles $\left(-80^{\circ} \mathrm{C}\right.$ and $\left.37^{\circ} \mathrm{C}\right)$. The empty AAV8 vector was also packaged, yielding the AAV8-Vector. After purifying and concentrating by ultrafiltration, the titrated virus yields ranged from $10^{12}$ to $10^{13}$ genomic copies $/ \mathrm{mL}$.

\section{Construction of AAV8-shRNA Expression Vector Targeting Mmp2}

Screening of shRNA sequences targeting mouse Mmp2 gene was performed in NIH-3T3 cells (ATCC). The sequences for the shRNA targeting were 5'-GGGAGATTCTCACTTTGATGA-3'. The shRNA-Mmp2 and the scrambled control shRNA sequences were respectively ligated into AAV8-shRNA shuttle vector to construct AAV8-shRNA-Mmp2 and AAV8-shRNA-scramble (labeled as shRNA-Mmp2 and shRNA-scramble) for AAV8 packaging. They were packaged according to the procedure described in "Construction of AAV8-Mmp2 Overexpression Vector," providing titrated yields of virus that ranged from $10^{12}$ to $10^{13}$ genomic copies $/ \mathrm{mL}$.

\section{Method for AAV8 Injection}

Injection was performed under a Zeiss (Gottingen, Germany) operating microscope in the right eye of each mouse. Mice were narcotized with a mixture of ketamine $(72 \mathrm{mg} / \mathrm{kg}$ ) and xylazine $(4 \mathrm{mg} / \mathrm{kg})$ by i.p. injection. Hydroxymethyl cellulose (2.5\%) was applied to avoid desiccation before surgery. Posterior Tenon's capsule was entered along the eyeball radian from 14 to $18 \mathrm{~mm}$ away from the corneal limbus and eyeball equatorial axis, followed by delivery of $5 \mu \mathrm{L}$ of virus. Eyes were covered with $0.2 \%$ polyacrylic acid every day for 3 days after surgery. 


\section{Experimental Design}

Wild-type male mice eyeballs were used in this study if their interocular refraction difference between the right and left eyes was $<3.00$ diopter and appeared to be uninjured and free of infection. The detailed flow chart of the experimental design is shown in Supplemental Figure S2.

The time course of MMP-2 expression during myopia development was determined in 3-week-old wild-type mice. They were randomly divided into two groups: composed of mice that underwent monocular FD treatment for either 2 days, 1 week, or 2 weeks and age-matched mice with no FD (labeled as control).

To assess if the AAV8 could transfect the sclera effectively, sub-Tenon's capsule injection of AAV8-GFP was first performed in the 3-week-old mice. The eyes injected with AAV8-Vector served as the control. GFP was detected after 1 week of AAV8-GFP or AAV8-Vector injection.

To determine whether scleral MMP-2 up-regulation could induce myopia, 3-week-old wild-type mice $(n=41)$ were randomly divided into three groups: 14 were respectively injected with either AAV8-Mmp2 or AAV8-Vector and another 13 noninjected mice served as the control (labeled as AAV8-Mmp2, AAV8-Vector, and control, respectively). Ocular measurements were recorded before AAV8 injection and 4 weeks later (week 3 and 7).

To determine the effect of scleral MMP-2 knockdown on the normal refraction development, 3-week-old wild-type mice $(n=44)$ were randomly assigned to three groups: 15 mice were injected with either shRNA-Mmp2 or shRNAscramble and another 14 noninjected mice served as the control (labeled as shRNA-Mmp2, shRNA-scramble, and control, respectively). Ocular measurements were recorded before AAV8 injection and 4 weeks later (week 3 and 7).

To determine the effect of scleral MMP-2 knockdown on FDM development, 3-week-old wild-type mice $(n=60)$ were randomly assigned to three groups: 23 mice were injected with either shRNA-Mmp2 or shRNA-scramble, and after a week FDM was initiated for the next 4 weeks (labeled as shRNA-Mmp2 + FD and shRNAscramble + FD). Another 14 noninjected mice served as controls (labeled as Control + FD). Ocular measurements were measured before and 4 weeks after FDM (week 4 and 8).

To determine the effect of fibroblast-specific Mmp2 deletion on the normal refractive development, ocular measurements were tracked from postnatal day $(\mathrm{P}) 28$ to $\mathrm{P} 70\left(4,6,8\right.$, and 10 weeks) in $12 \mathrm{S100a4^{cre }} \mathrm{Mmp}^{\text {fl/fl }}$ and $25 M m p 2^{f l f l}$ mice. To determine the effect of S100a $4^{\text {cre }} \mathrm{Mmp} 2^{\text {fllfl }}$ on FDM development, 16 $S 100 a 4^{\text {cre }} \mathrm{Mmp} 2^{f l / f}$ and $18 \mathrm{Mmp} 2^{f / f l}$ mice 4 weeks old were subjected to 2 weeks of FD (labeled as $S 100 a 4^{\text {cre }} M m p 2^{f l}$ $f l+$ FD and $M m p 2^{f / f l}+$ FD), and ocular measurements were performed before and 2 weeks after FD was initiated (4 and 6 weeks).
RNA Extraction, cDNA Preparation, and Quantitative Real-Time PCR

At the end of various treatment periods, the eyes were isolated. The sclera, cornea, and lens were dissected and homogenized separately in a ball mill, and total RNA was extracted using the RNeasy fibrous tissue minikit (Qiagen, Valencia, CA) according to the manufacturer's instructions. The total retinal RNA was extracted by using TRIzol reagent (Invitrogen, Carlsbad, CA) according to the manufacturer's protocol. After being treated with RQ1 RNaseFree DNase (Promega, Madison, WI), the RNAs of sclera, cornea, and retina were subjected to reverse transcription with random primers and Moloney Murine Leukemia Virus Reverse Transcriptase (Promega) to synthesize respective cDNAs. ${ }^{37}$ RT-PCR was performed by using the specific primers and Power SYBR Green PCR Master Mix (Applied Biosystems, Carlsbad, CA) on an ABI 7500 Real-Time PCR system. The expression of each target mRNA relative to $18 \mathrm{~S}$ $r R N A$ (sclera, retina, cornea, lens) was determined by using threshold cycle values and the ${ }^{\Delta \Delta} \mathrm{Ct}$ method. ${ }^{38}$ The identity of these above-mentioned primer sequences and primers used specifically for $S 100 a 4^{c r e} M m p 2^{f l f l}$ mice were confirmed with RT-PCR analysis and are provided in Table 1.

\section{Western Blot Analysis}

Sclerae from different mice were homogenized in $150 \mu \mathrm{L}$ of ice-cold radiommunoprecipitation assay buffer (Beyotime, Shanghai, China) supplemented with $1 \mathrm{mmol} / \mathrm{L}$ phenylmethylsulfonyl fluoride (Beyotime) by using a ball mill, followed by sonication for approximately 30 to 60 seconds for complete tissue lysis. After being centrifuged at 13,000 $\times g$ for 10 minutes at $4{ }^{\circ} \mathrm{C}$, the supernatant was aspirated and placed in a fresh tube on ice. The protein content was determined by using an Enhanced BCA Protein Assay Kit (Beyotime).

Equal amounts of proteins $(50 \mu \mathrm{g})$ from each sample were loaded onto $10 \%$ SDS-PAGE gel, separated by electrophoresis, and electrotransferred onto a nitrocellulose membrane (Millipore, Billerica, MA). After blocking with 5\% nonfat milk for 2 hours at room temperature, the membrane was incubated with primary antibodies overnight at $4^{\circ} \mathrm{C}$. Primary antibodies against collagen type $1, \alpha 1$ (Col1 $\alpha 1$; dilution 1:2000; ab88147; Abcam, Cambridge, MA), MMP-2 (dilution 1:1000; ab37150; Abcam), and $\alpha$-tubulin (dilution 1:2000; ab52866; Abcam) were used. After washing thrice with tris-buffered saline with Tween-20 $[10 \mathrm{mmol} / \mathrm{L}$ Tris-HCl (pH 8.0), $150 \mathrm{mmol} / \mathrm{L} \mathrm{NaCl}$, and $0.1 \%$ Tween-20] for $5 \mathrm{mi}-$ nutes each, a membrane was incubated with dilution 1:2000 IRDye $800 \mathrm{CW}$ goat anti-rabbit IgG (926-32211; Odyssey, Lincoln, NE) or dilution 1:2000 IRDye $800 \mathrm{CW}$ goat antimouse IgG (926-32210; Odyssey) antibodies for 2 hours at room temperature. The membrane was again washed in trisbuffered saline with Tween- 20 thrice as before, followed by 
Table 1 PCR Primers Used in This Study

\begin{tabular}{|c|c|c|}
\hline Primer name & Forward primer & Reverse primer \\
\hline \multicolumn{3}{|c|}{ Primers for RT-PCR } \\
\hline $18 \mathrm{~S}$ & $5^{\prime}-$ CGGACACGGACAGGATTGAC-3' & $5^{\prime}$-GTTCAAGCTGCCCGTCTCCTCATC-3' \\
\hline$M M P-2-C K O$ & $5^{\prime}-\mathrm{TGCTGAAAGATACCCTCAAGAAGAT-3^{ \prime }}$ & $5^{\prime}$-TATGTGATCTGGTTCTTGTCCCAC-3' \\
\hline Col1 $\alpha 1$ & $5^{\prime}$-GAGAGCGAGGCCTTCCCGGA-3' & $5^{\prime}$-GGGAGCCAGCGGGACCTTGT-3' \\
\hline \multicolumn{3}{|c|}{ Primers for genotype identification of $M M P 2^{f / f}$ and $S 100 a 4^{\text {Cre }}$} \\
\hline Null & $5^{\prime}$-ATACCAGGCACATAGAGGAGAGGTC- $3^{\prime}$ & 5'-GCTGGTTGTAGTTTGGGGCAGA-3' \\
\hline S100a4 ${ }^{\text {re }}$ & $5^{\prime}$-GGATAGAGCACTTAAAATGGGGA-3' & 5'-GTGAAACAGCATTGCTGTCACTT-3' \\
\hline
\end{tabular}

densitometric analysis of the protein bands by using Image $\mathbf{J}$ version 1.48 software (NIH, Bethesda, MD). The values were normalized to corresponding loading control, $\alpha$-tubulin. All Western blot analyses shown are representative of at least three independent experiments.

\section{Immunofluorescence and Confocal Microscopy}

Four-week-old S100a4 $4^{\text {cre }} R O S A^{m T / m G}$ and $R O S A^{m T / m G}$ mice were sacrificed, and the eyes were isolated. The eyecups were then fixed in freshly prepared $4 \%$ paraformaldehyde for 20 minutes at room temperature and dehydrated in a gradient of 10\% (2 hours), 20\% (2 hours), and $30 \%$ (overnight) sucrose solutions at $4{ }^{\circ} \mathrm{C}$. The eyecups were embedded in Neg 50 Frozen Section Medium (Thermo Fisher Scientific, Waltham, MA), and the globes were then frozen in liquid nitrogen and stored at $-80^{\circ} \mathrm{C}$ until sectioned. The eyecups were cut into $12-\mu \mathrm{m}$-thick sections by using a freezing microtome. After rinsing the slides thrice in phosphate-buffered saline for 5 minutes each, they were then stained with DAPI (Vector Laboratories, Burlingame, CA). Neither tdTomato (red) nor GFP (green) required immunostaining for visualization. The frozen sections were visualized and photographed with a Zeiss LSM 710 confocal microscope (Zeiss).

To detect the GFP distribution in the sclera after 1 week of AAV8-GFP or AAV8-Vector injection, mice were sacrificed and the eyes were isolated. The eyecups were dissected to generate scleral flat-mount, then the specimens were fixed in freshly prepared $4 \%$ paraformaldehyde for 20 minutes at room temperature. After rinsing the slides thrice in phosphate-buffered saline for 5 minutes each, they were then stained with DAPI. The flat-mount sclera was visualized and photographed with a Zeiss LSM 710 confocal microscope.

\section{Statistical Analysis}

All data were analyzed by using statistical software (GraphPad Prism Software, version 7.0; GraphPad Inc., La Jolla, CA). A normality test (Shapiro-Wilk normality test) analyzed the distribution of all data sets. For comparison of two groups, the differences were assessed by using parametric tests such as $t$-test (for normality of distributed data), nonparametric $U$-test, or Wilcoxon matched-pairs signed rank test. Multiple comparisons were performed by using one-way analysis of variance with Bonferroni's post hoc test (for normality of distributed data) or Kruskal-Wallis nonparametric with Dunn's post hoc test. Two-way analysis of variance for repeated measurements was used to compare the biometric measurements of the normal refractive development in the $S 100 a 4^{c r e} M m p 2^{f l / f l}$ and $M m p 2^{f / f l}$ mice, and the effect of genotype on time-dependent refractive development was evaluated with the Bonferroni post hoc test. Values of $P<0.05$ were considered statistically significant.

\section{Results}

Scleral MMP-2 Expression Profile during Myopia Development

To evaluate the association between scleral MMP-2 expression and myopia, 3-week-old male wild-type C57BL/6 mice were subjected to FD for either 2 days, 1 week, or 2 weeks during which time scleral MMP-2 expression levels were measured. After 2 days of FD, the mRNA expression level of Mmp2 in the sclera of FD-T eyes was significantly higher than in FD-F $(P<0.05)$ and control eyes $(P<0.05)$ (Figure $2 \mathrm{~A})$, whereas refraction was not statistically significant $(P=0.66)$ (Figure $2 \mathrm{~B})$. After 1 week of $\mathrm{FD}$, a myopic shift in refraction occurred $(P<0.001)$ (Figure $2 \mathrm{C})$. This myopic shift was accompanied by a further increase in Mmp2 mRNA levels $(P<0.01)$ (Figure 2A) compared with the control eyes. After 2 weeks of FD, the mice became more myopic than that observed after 1 week $(P<0.01)$ (Figure 2D) with longer AL $(P<0.05)$ (Figure $2 \mathrm{E}$ ) in FD-T eyes. Although the VCD of FD-T eyes increased slightly relative to FD-F eyes (Figure 2F), the difference was not statistically significant $(P=0.07)$. After 2 weeks of FD, the Mmp2 mRNA level was significantly higher than in FD-F eyes $(P<0.05)$ and control eyes $(P<0.05)$ (Figure $2 \mathrm{~A})$. 


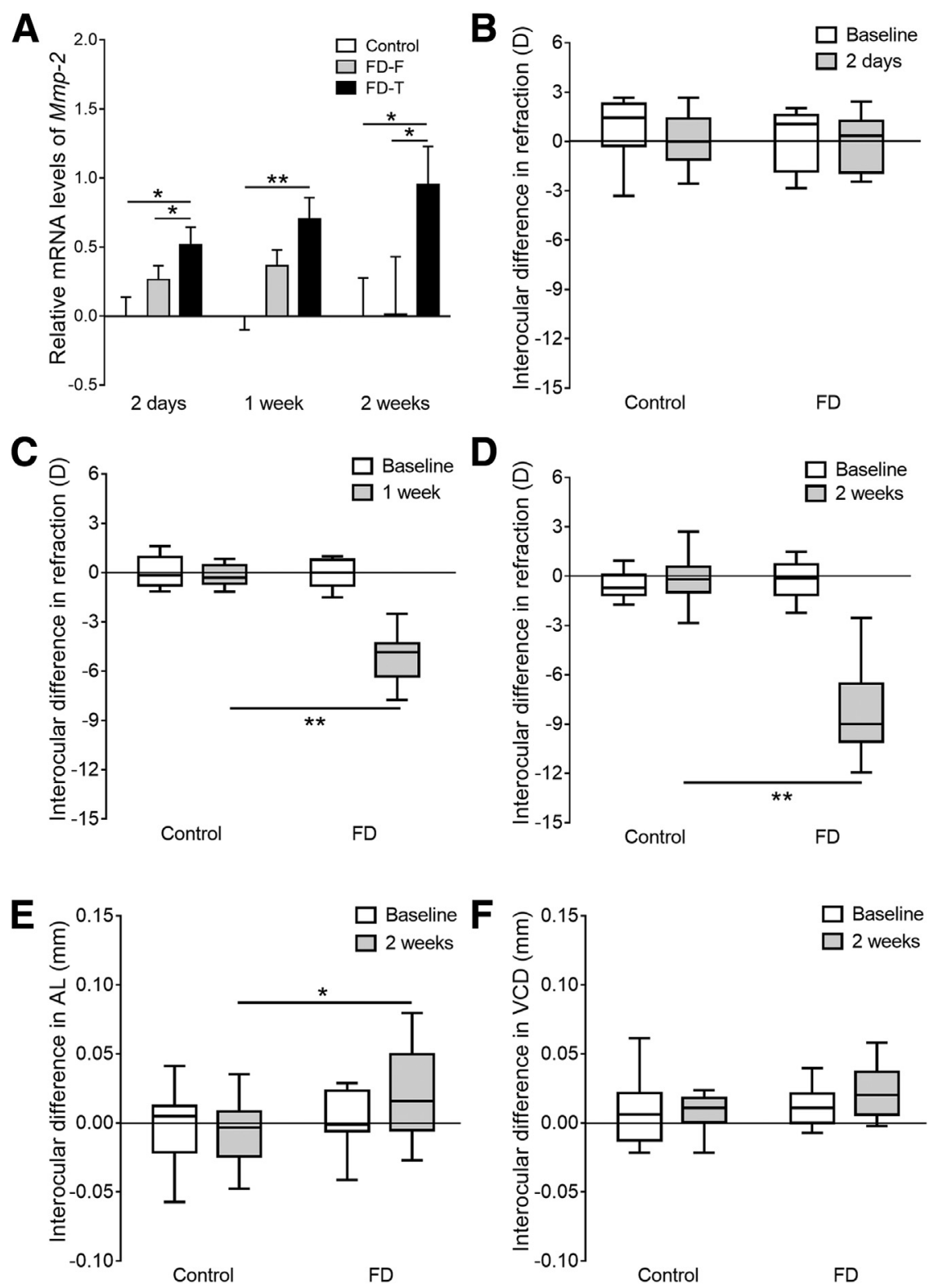

Figure 2 Form deprivation (FD) induced rises in scleral matrix metallopeptidase (MMP)-2 expression. Right eye of each mouse in FD groups were subjected to FD (FD-T), and the left eyes served as the fellow controls (FD-F). Data from age-matched mice without FD induction are also presented for comparison (Control). A: Time course quantification from RT-PCR analysis of scleral Mmp2 mRNA levels after 2 days, 1 week, and 2 weeks of FD; mRNA expression levels of Mmp2 were normalized to those of Control. B: After 2 days of $F D$, no significant myopia was induced. C: After 1 week of FD, significant myopia was induced. D-F: After 2 weeks of FD, the FD-T eyes become increasingly myopic, accompanied by axial length $(\mathrm{AL})$ elongation (E), a slight increase in vitreous chamber depth (VCD) was observed in the FD-T eyes (F), but there was no significant difference; the effects induced by FD are shown as interocular differences, that is, the difference between FD-T/ right eyes and FD-F/left eyes (FD-T/right eyes FD-F/left eyes). Data are expressed as means \pm SEM (A). Box plot diagrams showing the distribution of the data. Line within the box indicates median; bars, range. $n=8$ mice for each group at each time point (A); $n=8$ control mice (B); $n=12$ FD mice (B); $n=10$ control mice (C); $n=13 \mathrm{FD}$ mice $(\mathbf{C}) ; n=16$ control mice $(\mathbf{D}-\mathbf{F})$; $n=15 \mathrm{FD}$ mice $(\mathbf{D}-\mathbf{F}) .{ }^{*} P<0.05,{ }^{* *} P<0.01$. For the comparison of the Mmp2 expression between FD-T and Control groups, unpaired $t$-test was used; for the comparison of the Mmp2 expression difference between FD-T and FD-F groups, paired $t$-test was used (A). For the comparison of the interocular differences in refraction, $\mathrm{AL}$, and VCD between the FD and Control groups, unpaired $t$-test or nonparametric $U$-test was used $(\mathbf{B}-\mathbf{F})$. D, diopter.
Like most other MMPs, MMP-2 was synthesized and secreted as an inactive latent form and activated by enzymatic cleavage in the extracellular environment. Accordingly, both the latent and active forms of MMP-2 in sclera were measured. In the FD-T eyes, the latent and active forms of MMP-2 levels in sclera were markedly higher than in the control and FD-F eyes after 2 days $(P<0.05)$ (Supplemental Figure S3, A and C) and 2 weeks $(P<0.05)$ (Supplemental Figure S3, B and D) of FD, which was consistent with the higher Mmp2 mRNA expression that accompanied FD-induced scleral remodeling. After 2 weeks of FD, the scleral Coll $\alpha 1$ accumulation in FD-T eyes was significantly lower than in the control $(P<0.05)$ and FD-F $(P<0.05)$ eyes (Supplemental Figure S3, B and E).

\section{Scleral MMP-2 Up-Regulation Induces Myopia}

To determine the efficacy of AAV8-mediating gene transfer in sclera, the GFP in the flat-mount sclera was examined after 1 week of AAV8-GFP or AAV8-Vector injection. GFP was apparent in the whole sclera in the AAV8GFP-injected eyes; however, no GFP was detected in the AAV8-Vector-injected eyes (Supplemental Figure S4).

To determine whether scleral MMP-2 up-regulation affected refractive development in wild-type mice, AAV8Mmp2 was injected into sub-Tenon's capsule of the right eyes. The scleral Mmp2 gene expression level at 4 weeks after AAV8-Mmp2 injection was significantly greater than that in the AAV8-Vector-injected eyes $(P<0.001)$ (Figure 3A). Similarly, both the latent $(P<0.05)$ and active $(P<0.05)$ 

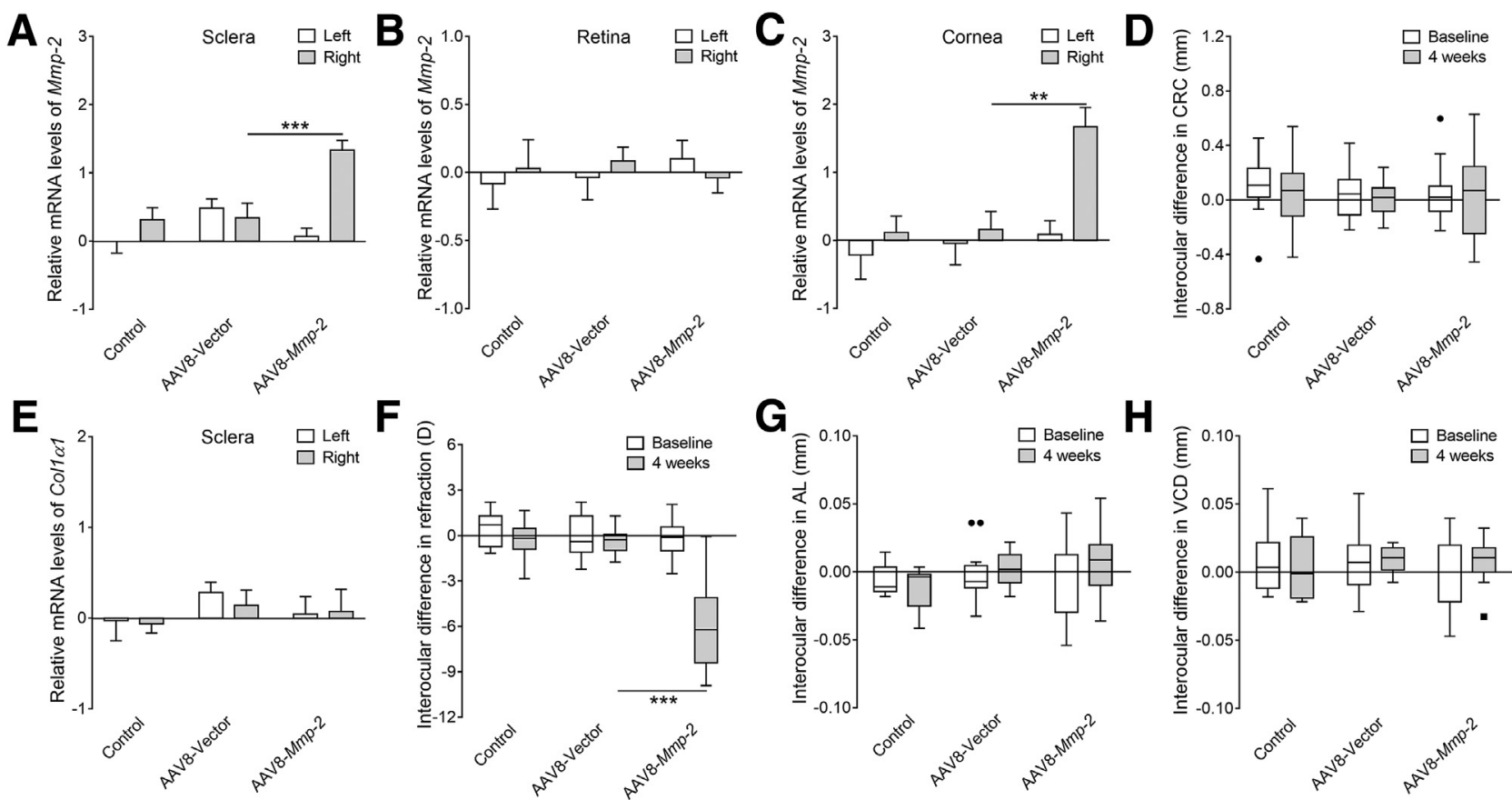

Figure 3 Selective promotion of MMP2 gene expression by adeno-associated virus serotype 8 (AAV8)-Mmp2 injection induces myopia. Right eye of each mouse (Right) was injected with AAV8-Mmp2 or AAV8-Vector; the left eye (Left) served as the fellow control. Data from age-matched mice with no treatment are also presented for comparison (Control). A-C: RT-PCR analysis of Mmp2 mRNA expression levels in sclera, retina, and cornea. D: No significant difference was observed in interocular differences in corneal radius curvature (CRC). E: RT-PCR analysis of Col1a1 mRNA expression in sclera. Gene expression levels in parts $\mathbf{A}-\mathbf{C}$ and $\mathbf{E}$ were normalized to those of Left of Control. F: AAV8-Mmp2-injected eyes become significantly myopic compared with the AAV8-Vector injected eyes. $\mathbf{G}$ and $\mathbf{H}$ : No significant differences in the interocular differences in axial length (AL) and vitreous chamber depth (VCD) were observed between the AAV8-Vector and AAV8-Mmp2 groups. Data are expressed as means \pm SEM ( $\mathbf{A}-\mathbf{C}$ and $\mathbf{E})$. Box plot diagrams showing the distribution of the data. Line within the box indicates median; bars, range; black dots, outliers. $n=7$ control mice $(\mathbf{A}-\mathbf{C}$ and $\mathbf{E}) ; n=8$ AAV8-vector mice $(\mathbf{A}-\mathbf{C}$ and $\mathbf{E}) ; n=8$ AAV8-Mmp-2 mice $(\mathbf{A}-\mathbf{C}$ and $\mathbf{E}) ; n=13$ control mice $(\mathbf{D}$ and $\mathbf{F}-\mathbf{H}) ; n=14$ AAV8-vector mice (D and $\mathbf{F}-\mathbf{H}) ; n=14$ AAV8-Mmp-2 mice $(\mathbf{D}$ and $\mathbf{F}-\mathbf{H})$. ${ }^{* *} P<0.01$, ${ }^{* * *} P<0.001$. Differences among Control, AAV8-Vector, and AAV8-Mmp2 groups were assessed by using one-way analysis of variance with Bonferroni's post hoc test (for normality distributed data) or Kruskal-Wallis nonparametric with Dunn's post hoc test. D, diopter.

forms of MMP-2 in sclera were markedly greater in the AAV8-Mmp2-injected eyes than in the AAV8Vector-injected eyes (Supplemental Figure S5, A and B). No significant difference in retinal Mmp2 mRNA expression was observed between the AAV8-Mmp2- and AAV8Vector-injected eyes $(P=0.63)$ (Figure 3B). Although a greater corneal Mmp2 mRNA expression level was observed in AAV8-Mmp2-injected eyes than in the AAV8Vector-injected eyes $(P<0.01)$ (Figure $3 \mathrm{C})$, no significant difference in the CRC was observed $(P=0.95)$ (Figure 3D), which suggested that elevation of corneal MMP-2 expression did not have a significant effect on refraction.

The effect of MMP-2 up-regulation on scleral Coll $\alpha 1$ expression was evaluated because type I collagen accounts for approximately $90 \%$ of the scleral total dry weight. No significant difference in scleral Collal mRNA expression was observed between AAV8-Mmp2- and AAV8Vector-injected eyes $(P=0.80)$ (Figure 3E). However, its protein expression was $28 \%$ lower in AAV8-Mmp2-injected eyes than in their noninjected fellow eyes $(P<0.01)$ (Supplemental Figure S5, A and C), whereas AAV-Vector injection had no significant effect on Coll $\alpha 1$ protein expression $(P=0.31)$ (Supplemental Figure S5, A and C).
Baseline measurements of all biometric variables were similar among the three groups and between eyes of individual mice in the same group. No significant differences in any of these variables were detected between the control and fellow eyes of all groups at any time. Thus, the interocular differences in refraction and axial components between injected eyes and their fellow eyes (injected/right eyes their fellow/left eyes) were used to assess the biometric changes after AAV8 injection. Data from control groups are also shown for comparison.

Scleral MMP-2 up-regulation resulted in a myopic shift in refraction in the AAV8-Mmp2-injected eyes 4 weeks after injection compared with AAV8-Vector-injected eyes $(P<0.001)$ (Figure 3F). However, $\mathrm{AL}(P=0.49)$ and VCD $(P=0.70)$ differences were not statistically significant (Figure 3, G and H). This disconnect between changes in refraction and ocular biometric invariance may be due to the limited resolving power of optical coherence tomograph to identify any biometric changes if they fell below the level of instrument detection. ${ }^{39}$

Taken together, scleral MMP-2 up-regulation induced a significant myopic shift in refraction but left $\mathrm{AL}$ and $\mathrm{VCD}$ unaltered. 
Scleral MMP-2 Knockdown Suppresses FDM

Development without Altering Normal Refractive Development

Four weeks after shRNA-Mmp2 injection, the scleral Mmp2 gene expression level was significantly lower than in shRNA-scramble-injected eyes $(P<0.001)$, (Figure 4A). However, no significant difference was observed in retinal Mmp 2 mRNA expression between the shRNA-Mmp2- and shRNA-scramble-injected eyes $(P=0.90)$ (Figure 4B). Although shRNA-Mmp2 injection lowered Mmp2 mRNA expression compared with shRNA-scramble-injected eyes $(P<0.01)$ (Figure $4 \mathrm{C}$ ), no significant difference in CRC was observed $(P=0.96)$ (Figure $4 \mathrm{D})$. No significant differences were observed in interocular differences in refraction $(P=0.20)$, AL $(P=0.29)$, and $\operatorname{VCD}(P=0.91)$ between the shRNA-scramble and shRNA-Mmp2 groups (Figure 4, E-G). Similarly, no significant difference was observed in scleral Collal mRNA expression between these two groups $(P=0.67)$ (Figure $4 \mathrm{H})$. Therefore, reducing the scleral Mmp2 expression level had no effect on either Collal gene expression level or normal refraction development in a normal visual environment.
To determine whether reduced scleral MMP-2 expression inhibited myopia development, FD was initiated 1 week after injecting either shRNA-Mmp2 or shRNA-scramble. After 4 weeks of FD, in the Control + FD group Mmp2 mRNA expression in FD-T eyes was significantly higher than in the FD-F eyes $(P<0.01)$, whereas $M m p 2$ mRNA expression in the FD-T eyes of the shRNA-Mmp2 + FD group was significantly lower than in the FD-T eyes of the shRNA-scramble + FD group $(P<0.001)$ (Figure 5A). These effects on gene expression levels paralleled higher MMP-2 active form levels in the FD-T eyes than in the FDF eyes of the Control + FD group $(P<0.05)$, whereas this increase was suppressed in the FD-T eyes of the shRNAMmp $2+$ FD group compared with the FD-T eyes of the shRNA-scramble + FD group $(P<0.05)$ (Supplemental Figure S6, A and B). After 4 weeks of FD, significant myopia was induced in all three groups, but inhibition of scleral MMP-2 expression by shRNA-Mmp2 inhibited the myopic refraction by approximately $23 \%$ compared with the shRNA-scramble + FD group $(P<0.05)$ (Figure 5 B). In parallel with the changes in refraction, $\mathrm{AL}$ elongations $(P<0.05)$ and increases in VCD $(P<0.05)$ were also significantly lower in the shRNA-Mmp2 + FD group than in
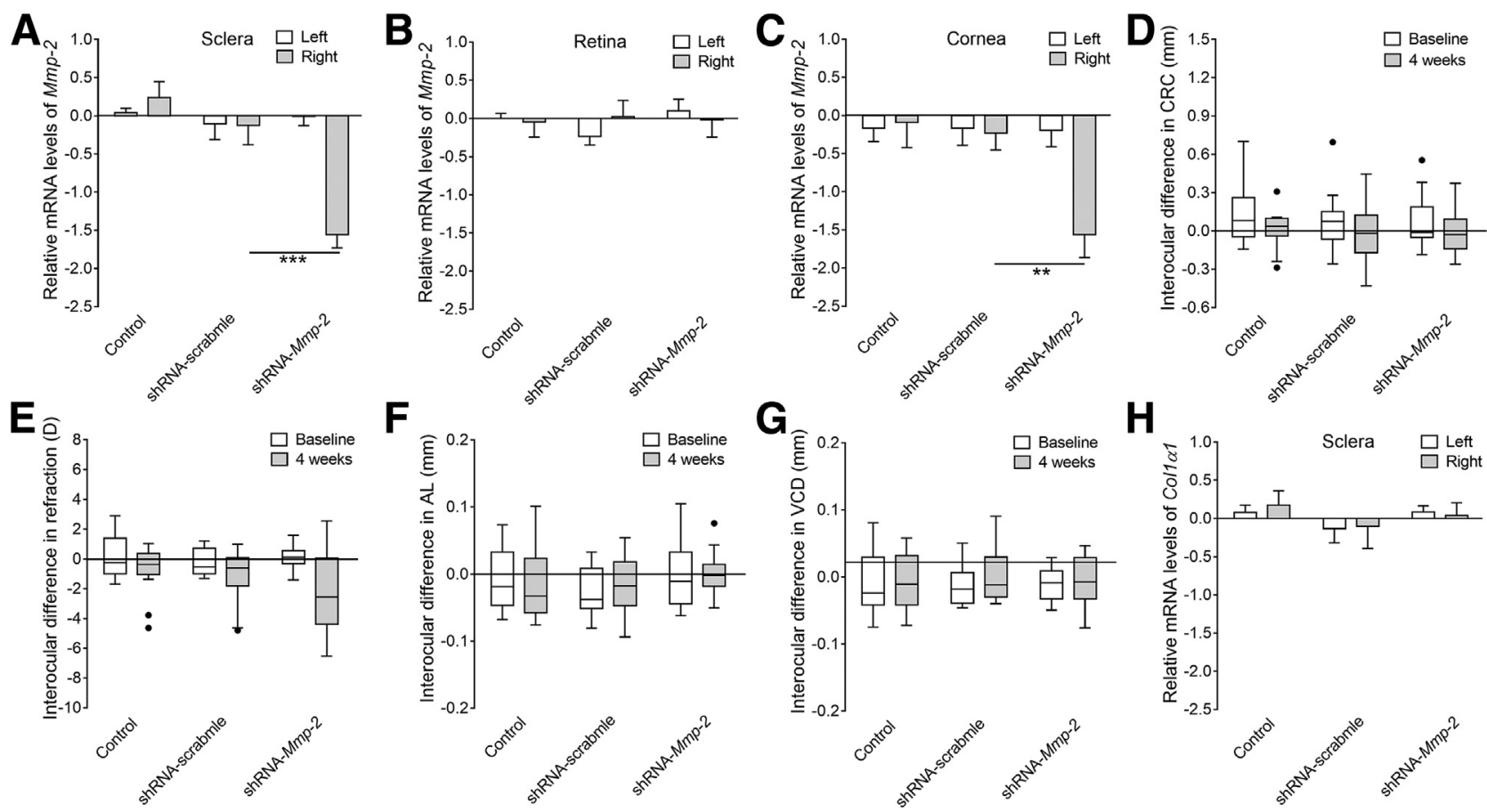

Figure 4 Effects of sub-Tenon's capsule injection of shRNA-Mmp2 on matrix metallopeptidase (MMP)-2 expression and ocular variables 4 weeks after injection. Right eye of each mouse (Right) was injected with shRNA-Mmp2 or shRNA-scramble; the left eye (Left) served as the fellow control. Data from agematched mice with no treatment are also presented for comparison (Control). A-C: RT-PCR analysis of Mmp2 mRNA expression in sclera, retina, and cornea. D-G: No significant differences were observed in interocular differences in corneal radius curvature (CRC), refraction, axial length (AL), and vitreous chamber depth (VCD) between the shRNA-Mmp2 and shRNA-scramble groups. H: RT-PCR analysis of Col1a1 mRNA expression in sclera. Data are expressed as means \pm SEM ( $\mathbf{A}-\mathbf{C}$ and $\mathbf{H})$. Gene expression levels in panels $\mathbf{A}-\mathbf{C}$ and $\mathbf{H}$ were normalized to those of Left of Control. Line within the box indicates median; bars, range; black dots, outliers. $n=6$ control mice $(\mathbf{A}-\mathbf{C}$ and $\mathbf{H}) ; n=7$ shRNA-scramble mice $(\mathbf{A}-\mathbf{C}$ and $\mathbf{H}) ; n=7$ shRNA-Mmp-2 mice $(\mathbf{A}-\mathbf{C}$ and $\mathbf{H}) ; n=14$ control mice (D-G); $n=15$ shRNA-scramble mice (D-G); $n=15$ shRNA-Mmp-2 mice (D-G). ${ }^{* *} P<0.01,{ }^{* *} p<0.001$. Differences among Control, shRNAscramble, and shRNA-Mmp2 groups were assessed by using one-way analysis of variance with Bonferroni's post hoc test (for normality distributed data) or Kruskal-Wallis nonparametric with Dunn's post hoc test. D, diopter. 

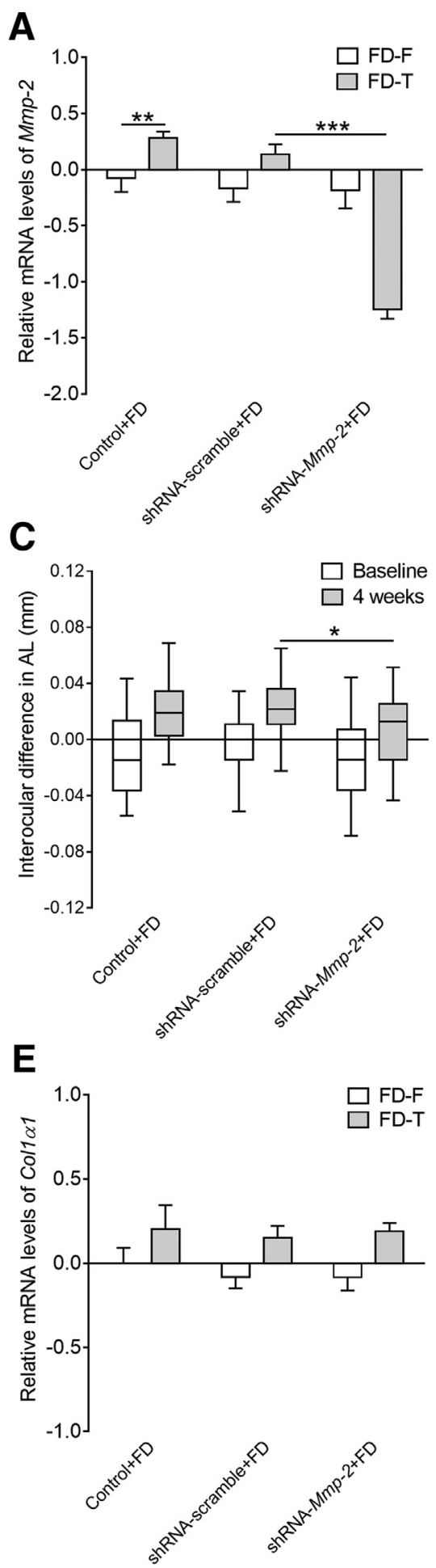

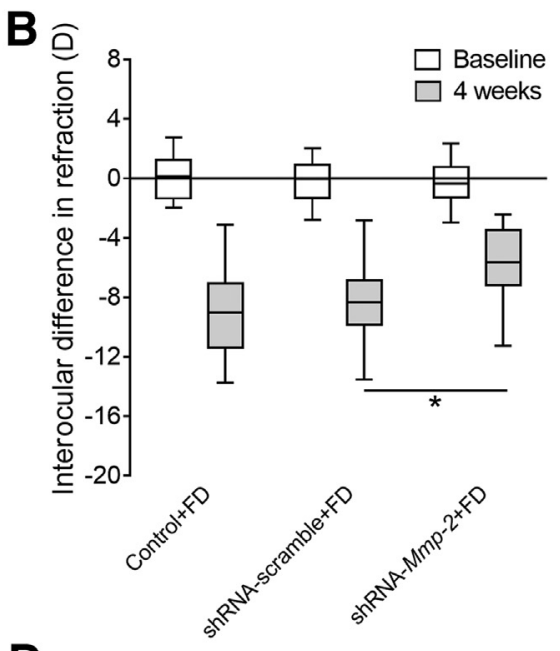

D

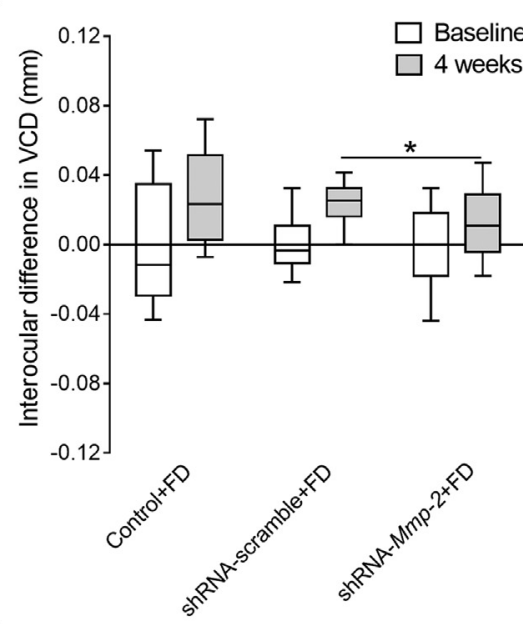

$\mathbf{F}$

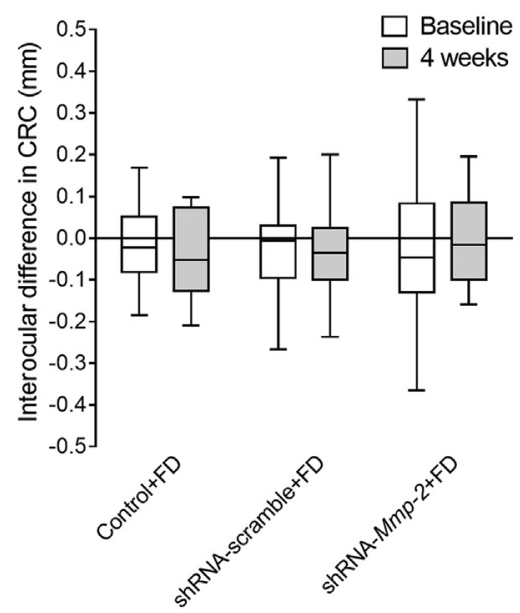

Figure 5 Scleral matrix metallopeptidase (MMP)-2 down-regulation inhibits form deprivation myopia (FDM) development. Right eye of each mouse was injected with shRNA-Mmp2 or shRNAscramble, and 1 week after injection FD was imposed for a subsequent 4 weeks (FD-T) and the left eyes (FD-F) served as the fellow controls; the form-deprived noninjected mice (Control + FD) served as the control. A: RT-PCR analysis of the scleral Mmp2 mRNA expression. B-D: The myopia induced in the shRNA-Mmp2 + FD group was less than in the shRNA-scramble $+F D$ group, which was accompanied by less axial length ( $\mathrm{AL}$ ) elongation and smaller increases in vitreous chamber depth (VCD). E: RT-PCR analysis of the scleral Col1a1 mRNA expression. F: No significant difference was observed in corneal radius curvature (CRC). Data are expressed as means \pm SEM (A and E). Gene expression levels in parts $\mathbf{A}$ and $\mathbf{E}$ were normalized to those of Left of Control + FD. Line within the box indicates median; bars, range. $n=9$ control mice (A and $\mathbf{E}$ ); $n=15$ AAV8-Vector mice ( $\mathbf{A}$ and $\mathbf{E}) ; n=15$ AAV8-Mmp-2 mice (A and $\mathbf{E}) ; n=14$ control mice (B-D and $\mathbf{F}) ; n=23$ AAV8-vector mice (B-D and $\mathbf{F}) ; n=23$ AAV8-Mmp-2 mice (B-D and $\mathbf{F})$. ${ }^{*} P<0.05$, one-way analysis of variance with Bonferroni's post hoc test; ${ }^{* *} P<0.01$, for Control + FD group: FD-F versus FD-T, paired $t$-test; ${ }^{* * *} P<0.001$. Differences among Control $+\mathrm{FD}$, shRNA-scramble, and shRNA-Mmp2 groups were assessed by using one-way analysis of variance with Bonferroni's post hoc test. Differences in the scleral Col1a1 mRNA expression were assessed by using Kruskal-Wallis nonparametric with Dunn's post hoc test. D, diopter. the shRNA-scramble + FD group (Figure 5, C and D). No significant difference in scleral Collal mRNA expression was observed between the shRNA-Mmp2 and shRNAscramble groups $(P=0.73)$ (Figure $5 \mathrm{E})$. Nevertheless, Col1 $\alpha 1$ protein expression in FD-T eyes was $25 \%$ lower than in the FD-F eyes of shRNA-scramble injected mice $(P<0.01)$, whereas this decline was not observed in the
FD-T eyes of the shRNA-Mmp2 + FD group $(P=0.09)$ (Supplemental Figure S6, A and C). In addition, no significant difference in the CRC was observed between the shRNA-Mmp $2+$ FD and shRNA-scramble + FD groups $(P=0.39)$ (Figure 5F). Therefore, reducing scleral MMP-2 expression suppressed both FDM development and the decline in Col1 $\alpha 1$ accumulation. 
Taken together, reducing scleral MMP-2 expression had no effect on normal refractive development but attenuated declines in Col1 $\alpha 1$ accumulation and FDM development.

\section{Fibroblast-Specific Mmp2 Deletion Lessens FDM}

Scleral fibroblasts are the predominant cell population in mammalian sclera and they are a primary source of MMP-2 expression. To confirm that reducing scleral MMP-2 contributed to a decline in myopia development, the CreloxP system was used to generate a novel fibroblast-specific Mmp2 deletion mouse model (Figure 1A).

To determine the distribution of S100a4 promoter driving Cre recombinase expression in the sclera, the GFP location in flat-mount sclera in $S 100 a 4^{\text {cre }} R O S A^{m T / m G}$ and $R O S A^{m T / m G}$ mice was assessed. Strong tdTomato fluorescence (red) was observed in all of the tissue (including the retina, cornea, sclera, and lens) in the $R O S A^{m T / m G}$ (Supplemental Figure S7A) and S100a4 ${ }^{\text {cre }} R O S A^{m T / m G}$ (Supplemental Figure S7B) mice. The GFP expression identified the location of Cre recombinase expression. It was distributed in the whole sclera of the S100a $4^{\text {cre }} R O S A^{m T / m G}$ mice (Supplemental Figure S7B) and also in the cornea and lens of the $S 100 a 4^{c r e}{ }^{R O S A} A^{m T / m G}$ mice (Supplemental Figure S7B).

Scleral MMP-2 knockdown efficiency obtained by fibroblast-specific Mmp2 deletion was significantly lower in the $S 100 a 4^{\text {cre }} M m p 2^{f / f l}$ mice than in the $M m p 2^{f / f l}$ mice $(P<0.05)$ (Figure 6A). The selectivity of this procedure in knocking down scleral Mmp2 gene expression was apparent from the lack of a difference between $M m p 2$ expression levels in the retina $(P=0.68)$ and cornea $(P=0.20)$ in the $S 100 a 4^{\text {cre }} \mathrm{Mmp}^{f / f l}$ and $M m p 2^{f / f l}$ mice (Figure 6A); however, the lens $\mathrm{Mmp} 2$ expression level in S100a4 ${ }^{\text {cre }} \mathrm{Mmp} 2^{f l / t}$ mice was significantly lower than in $M m p 2^{f / f l}$ mice $(P<0.01)$ (Figure 6A). No significant difference was found in scleral Collal mRNA expression between these two different genotypes $(P=0.23)$ (Figure $6 \mathrm{~B})$. Even though hyperopia developed in both genotypes from P28 to P70 (4 to 10 weeks) in a normal visual environment, the S100a $4^{\text {cre }} \mathrm{Mmp}^{\mathrm{flfl}}$ mice were less hyperopic than the $M m p 2^{f l f l}$ mice $(P<0.001)$ (Figure $\left.6 \mathrm{C}\right)$. Significant differences in refraction developed at P28 $(P<0.01)$, P42 $(P<0.05)$, P56 $(P<0.05)$, and P70 $(P<0.05)$ between the two genotypes (Figure $6 \mathrm{C}$ ). The AL increased more in the $S 100 a 4^{\text {cre }} \mathrm{Mmp}^{f l / f l}$ mice than in the $M m p 2^{f / f l}$ mice (Figure 6D). Significant differences in AL developed at P28 $(P<0.05)$, P42 $(P<0.01)$, P56 $(P<0.01)$, and P70 $(P<0.01)$ between the two genotypes (Figure 6D). The LT increased more in the $S 100 a 4^{\text {cre }} \mathrm{Mmp} 2^{f / f l}$ mice than in the $M m p 2^{f l / t}$ mice (Figure 6E). Significant differences in $\mathrm{AL}$ developed at P28 $(P<0.05)$, P42 $(P<0.01)$, P56 $(P<0.05)$, and $\mathrm{P} 70(P<0.05)$ between the two genotypes (Figure 6E).

To determine whether fibroblast-specific Mmp2 deletion could suppress myopia development, 4-week-old $S 100 a 4^{c r e} M m p 2^{f / f l}$ and $M m p 2^{f / f l}$ mice were subjected to FD for 2 weeks. Because the baseline values in the refraction, $\mathrm{AL}$, and LT components of these two genotypes were different (Figure 6, C-E), comparing raw data of these two
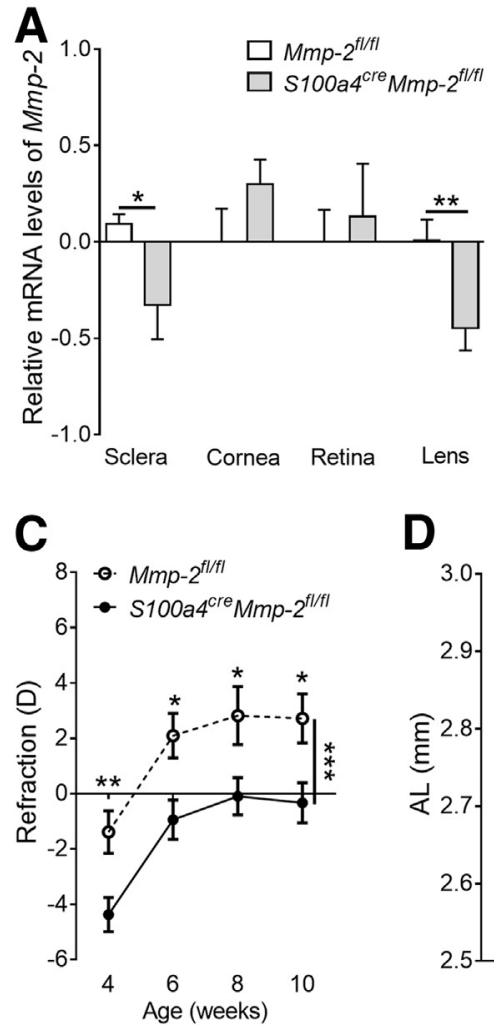

B
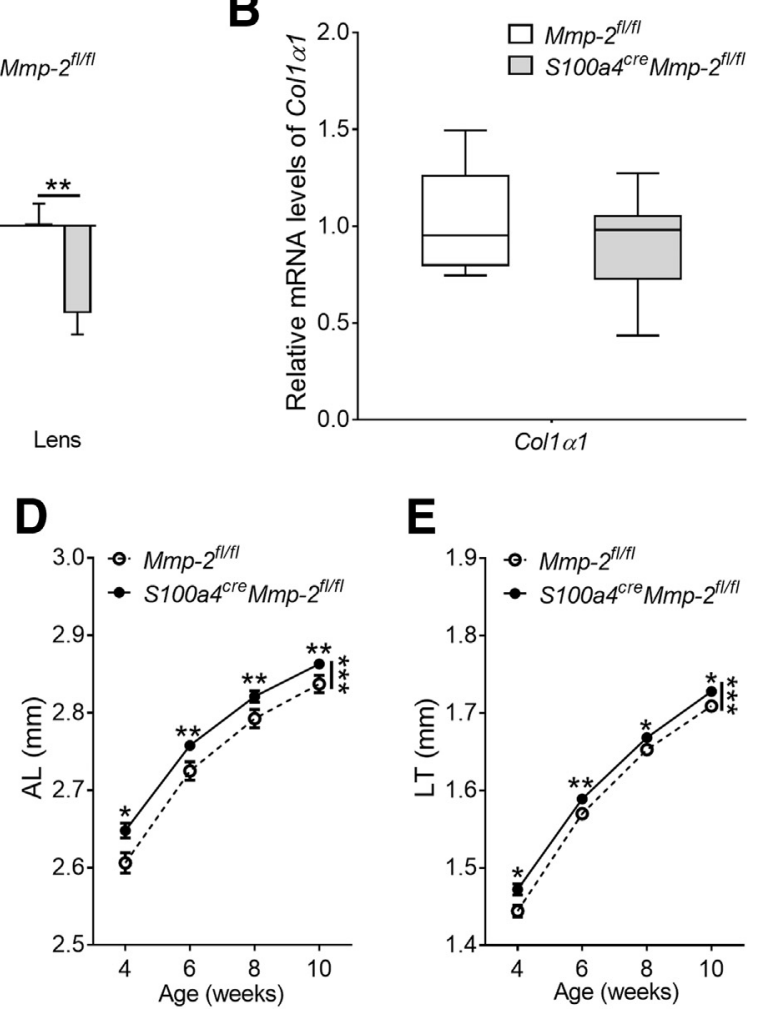

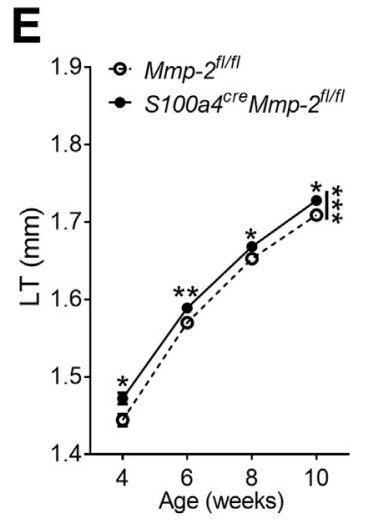

Figure 6 Retardation of refractive development in S100a4 ${ }^{\text {cre }} \mathrm{Mmp}^{\mathrm{fl} f \mathrm{fl}}$ mice. A: RT-PCR analysis of Mmp2 mRNA expression levels in sclera, cornea, retina, and lens in S100a4 $4^{\text {cre }} \mathrm{Mmp} 2^{f l f l}$ and $M m p 2^{f / f l}$ mice. B: RT-PCR analysis of Col1a1 mRNA expression levels in sclera. Gene expression levels in $\mathbf{A}$ and $\mathbf{B}$ were normalized to those of $M m p 2^{f / f f l}$. C-E: A significant myopic shift in refraction (C), greater axial length (AL; D), and greater lens thickness (LT; E) were observed in $S 100 a 4^{\text {cre }} M m p 2^{f l / f l}$ mice than in $M m p 2^{f l f l}$ mice at the indicated postnatal time points during normal refractive development. Data are expressed as means $\pm \operatorname{SEM}(\mathbf{A})$. Line within the box indicates median; bars, range. $n=125100 a 4^{\text {cre }} \mathrm{Mmp} 2^{f / f l}$ mice $(\mathbf{C}-\mathbf{E}) ; n=25 M m p 2^{f l / f l}$ mice $(\mathbf{C}-\mathbf{E}) ; n=12$ sclera (A); $n=5$ cornea (A); $n=5$ retina (A); $n=12$ lens $(\mathbf{A}) ; n=12 \mathrm{Mmp} 2^{f / f l}$ mice $(\mathbf{B}) ;$ $n=12$ S100ac ${ }^{\text {cre }} M m p 2^{f l f f l}$ mice (B). ${ }^{*} P<0.05$; ${ }^{* *} P<0.01$, determined by $U$-test or unpaired $t$-test $(\mathbf{A}$ and $\mathbf{B}) ;{ }^{* * *} P<0.001$, determined by two-way analysis of variance for repeated measurements for comparing the refraction and $A L$ in $S 100 a 4^{c r e} M m p 2^{f l / f l}$ and the $M m p 2^{f l f l}$ mice, the effect of genotype on time-dependent refractive development was evaluated by the Bonferroni post hoc test or Kruskal-Wallis nonparametric with Dunn's post hoc test (C-E). D, diopter. 


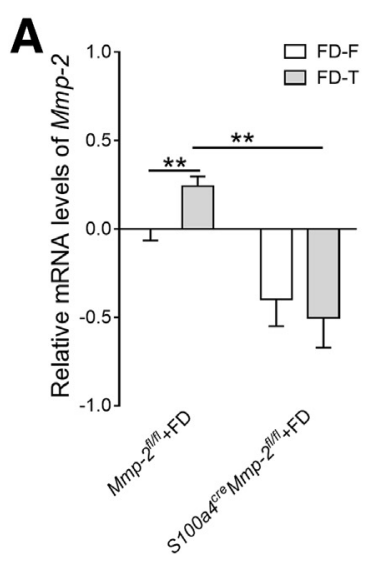

D

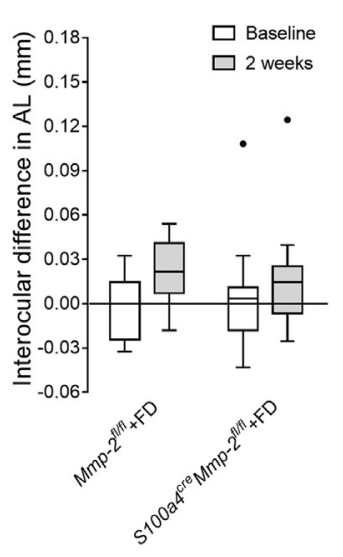

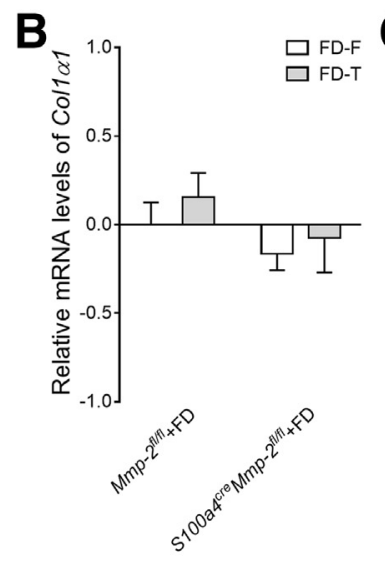

E

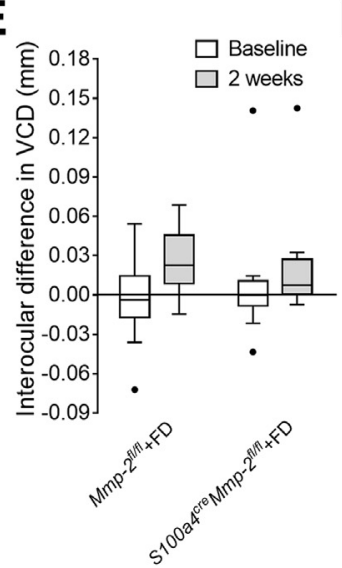

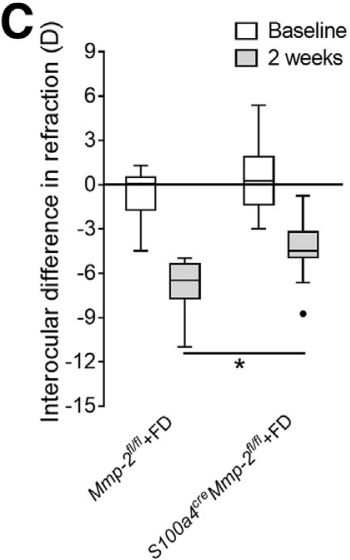

$\mathbf{F}$

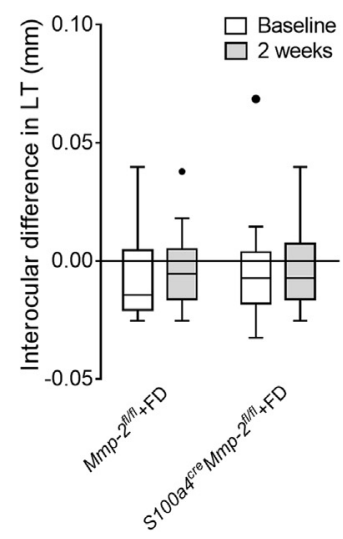

Figure 7 Fibroblast-specific Mmp2 deletion attenuates form deprivation myopia (FDM) $\left(\mathrm{S} 100 a 4^{\mathrm{cre}} \mathrm{Mmp} 2^{f l f l}\right)$ mice. A and B: RT-PCR analysis of scleral Mmp2 and Col1a1 mRNA expression of form-deprived eyes (FD-T) and fellow eyes (FD-F)

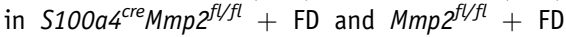
mice. Gene expression levels in panels $\mathbf{A}$ and $\mathbf{B}$ were normalized to those of FD-F of $M m p 2^{f l / f l}+F D$ mice. C: The myopia induced in the $S 100 a 4^{c r e} M m p 2^{f l f f l}+\mathrm{FD}$ group was approximately $27 \%$ lower than in the $M m p 2^{f / f l}+F D$ group. D-F: Interocular differences in axial length (AL; D), vitreous chamber depth (VCD;E), and lens thickness (LT; F) between S100a4 $4^{\text {cre }} \mathrm{Mmp} 2^{f l / f l}+\mathrm{FD}$ and $M m p 2^{f l f l}+F D$ groups were not statistically different. Data are expressed as means \pm SEM (A and $\mathbf{B})$. Line within the box indicates median; bars, range; black dots, outliers. $n=6 \mathrm{Mmp}^{f l / f l}+$ FD mice (A and B); $n=6 S 100 a 4^{c r e} M m p 2^{f l / f l}+F D$ mice $(\mathbf{A}$ and $\mathbf{B}) \cdot n=16 M m p 2^{f l f l}+\mathrm{FD}$ mice $(\mathbf{C}-\mathbf{F}) ; n=19$ S100a4 $4^{\text {cre }} \mathrm{Mmp2^{flfl }}+$ FD mice (C-F). ${ }^{*} P<0.05$, determined by nonparametric $U$-test; ${ }^{* * P}<0.01$, for Mmp $2^{f l f l}+$ FD group between FD-F versus FD-T, paired $t$-test or Wilcoxon matched-pairs signed rank test; for FD-T eyes between $S 100 a 4^{\text {Tre }} M m p 2^{f l y f l}+F D$ and $M m p 2^{f Y f l}+$ FD groups, unpaired $t$-test or $U$-test. $\mathrm{D}$, diopter. groups may be misleading. Thus, the interocular differences (FD-T - FD-F) in each mouse were instead used in the analysis.

After 2 weeks of FD, the mRNA expression $(P<0.01)$ (Figure 7A) and the latent $(P<0.05)$ and active $(P<0.05)$ forms of MMP-2 levels (Supplemental Figure S8, A and B) in sclera in FD-T eyes were significantly higher than in $M m p 2^{f / f l}$ mice but not in $S 100 a 4^{c r e} M m p 2^{f / f l}$ mice in comparison with respective levels in their FD-F eyes. The mRNA $(P<0.05)$ and active form $(P<0.05)$ of MMP-2 in FD-T eyes were significantly lower in $S 100 a 4^{\text {cre }} M m p 2^{f / f l}$ mice than in $M m p 2^{f / f l}$ mice (Figure 7A, Supplemental Figure S1, A and B). In addition, no significant difference was observed in Collal mRNA expression level of FD-T eyes between the two groups $(P=0.36)$ (Figure $7 \mathrm{~B}$ ), whereas its protein expression in FD-T eyes was significantly higher in the $S 100 a 4^{c r e} M m p 2^{f / f l}$ mice than in the $M m p 2^{f / f}$ mice $(P<0.05)$ (Supplemental Figure S8, A and C). Myopia induced in the $S 100 a 4^{c r e} M m p 2^{f l / f l}$ mice was $27 \%$ less than in the $M m p 2^{f / f l}$ mice $(P<0.05)$ (Figure $7 \mathrm{C}$ ). Both the AL elongation $(P=0.17)$ (Figure $7 \mathrm{D})$ and increase in VCD $(P=0.05)$ (Figure $7 \mathrm{E}$ ) were inhibited in $S 100 a 4^{c r e} M m p 2^{f / f l}$ mice slightly more than in the $M m p 2^{f / f l}$ mice, but these differences were not significant. No significant differences in the interocular difference in LT was observed between $S 100 a 4^{c r e} M m p 2^{f / f l}$ mice and $M m p 2^{f / f l}$ mice $(P=0.65)($ Figure $7 \mathrm{~F})$.
Taken together, reducing the scleral MMP-2 expression level by fibroblast-specific Mmp2 deletion suppressed both FDM and the declines in Col1 $\alpha 1$ accumulation relative to those in the $M m p 2^{f / f l}$ mice.

\section{Discussion}

This study demonstrates that there is a cause-and-effect relationship between MMP-2 up-regulation and myopia induced by FD in an experimental mouse model. Myopia initiation depended on increases in MMP-2 expression because MMP-2 expression levels were the same in the fellow and noninjected eyes in a normal visual environment, whereas FD induced up-regulation. Furthermore, the FD-induced increases in MMP-2 gene and protein expression levels preceded any detectable changes in refraction, AL, and VCD. Another finding consistent with this notion is that MMP-2 upregulation resulting from AAV8 gene transfer induced myopia, whereas declines in MMP-2 expression induced by shRNA suppressed FDM. This inhibitory effect on FDM was confirmed by showing that in $S 100 a 4^{\text {cre }} \mathrm{Mmp} 2^{f / f l}$ mice this response was also suppressed to the same extent as in the MMP2 shRNA-injected mice. These results agree with a prior study showing that MMP-2 is a viable target to inhibit myopia development. ${ }^{40}$ Despite all of the evidence supportive of a cause-and-effect relationship between increases in 
MMP-2 expression levels and myopia, it is still possible that there is an unknown confounder inducing in parallel these responses to form deprivation.

However, measuring the biometric variables accompanying changes caused by FD is a daunting task in mice because of their small eyes. This limitation makes it difficult to resolve differences, whereas it was possible to identify significant changes in the larger eyes of other experimental models. ${ }^{41}$ Our inability to detect significant changes in AL and VCD shown in Figures 2D, 3, G and H, and 5, E and F, is possibly attributable to the inability of optical coherence tomograph methods to resolve small changes in AL and VCD when the refraction change is small in mice.

MMP-2 involvement in mediating myopia development is based on previous studies showing that its up-regulation is associated with declines in scleral biomechanical strength and augmentation of this condition. ${ }^{10}$ Because selective MMP-2 inhibitors were unavailable, it was previously not possible to determine whether there was a cause-and-effect relationship between MMP-2 up-regulation and initiation as well as promotion of myopia development. However, we were able to selectively increase and decrease MMP-2 expression by manipulating its gene and protein expression levels. Specifically, MMP-2 up-regulation reduced type I collagen accumulation and induced myopia development, whereas declines in MMP-2 expression obtained by injecting Mmp2 gene shRNA function hindered myopia development. Similarly, in a conditional fibroblast Mmp2 deletion system a decline in scleral MMP-2 expression had effects similar to those obtained with the aforementioned shRNA system. Therefore, MMP-2-induced increases in collagen degradation underlying myopia appear to precede declines in scleral biomechanical strength identified in human sclera and other experimental animal models of this condition.

Even though genetic manipulations in mice identified several genes, including Egrl, Irbp, A2AR, and $\operatorname{Rar}^{42-45}$ whose expression modulation affects refractive development by using body-wide gene knockout mice, the identity of the cell type in which such a change occurred could not be determined. To deal with this limitation, the present study used a fibroblast-specific Mmp2 knockout model to determine the contribution made by scleral MMP-2 upregulation to myopia development. Using such a fibroblastspecific knockout could possibly clarify if MMP-2 release by other cell types besides fibroblasts affects myopia progression. ${ }^{46}$ However, it was not possible to resolve the contribution made by fibroblast MMP-2 expression to modulating myopia because the knockdown efficiency of Mmp2 expression in the fibroblast conditional knockout was relatively small. Another hindrance is that it is uncertain whether expression of MMP-2 by other cell types masked the effects of gain and loss of fibroblast-derived MMP-2 function on FDM development.

To the best of our knowledge, this is the first time a fibroblast-specific gene knockout mouse was developed with the intent to determine the contributory role of a single gene on refractive development and FDM progression. Even though our results indicate that increases in MMP-2 expression contribute to rises in type I collagen degradation and promotion of myopia development, in some cases the changes in MMP-2 expression and myopia progression do not correspond with one another. This occurred after shRNA-Mmp2 injection in which the active form of MMP-2 declined by $54 \%$, whereas myopia development declined by just 23\% (Supplemental Figure S6B and Figure 5B). Nevertheless, there is no reason to expect that there would be a one-to-one correspondence between changes in mRNA expression levels and FDM. Another consideration is that the lack of correspondence could be due to the involvement of other proteases besides MMP-2 inducing changes in myopia development. ${ }^{19}$ In the $S 100 a 4^{\text {cre }} \mathrm{Mmp} 2^{f / / f}$ mice, the active form of MMP-2 in sclera declined by $20 \%$, whereas myopia development declined by $27 \%$ (Supplemental Figure S8B and Figure 7C). However, this correspondence could be fortuitous because it is possible that there are other cell types besides fibroblasts in which Mmp2 gene expression is left unaltered by fibroblast-specific Mmp2 deletion. In addition, there could be compensatory effects resulting from up-regulation of other proteases induced by FD contributing to scleral remodeling. Another question pertains to the limited MMP-2 knockdown efficiency obtained in $S 100 a 4^{c r e} \mathrm{Mmp} 2^{f / f l}$ mice. One possibility for such a low efficiency could be due to limited fibroblast expression of the S100a4 promoter driving Cre recombinase expression.

\section{Conclusions}

This study used gain and loss of MMP-2 function strategies to confirm that changes in scleral MMP-2 expression directly contribute to modulating myopia in mice. The results show that increases in MMP-2 expression precede increases in FDM that stem from increases in both type I collagen degradation and presumably ECM remodeling. Because MMP-2 up-regulation had effects that were opposite to those caused by inhibiting MMP-2 expression, this result confirms that a cause-and-effect relationship exists between changes in MMP-2 expression and myopia in mice. These findings point to the possibility that developing selective MMP-2 inhibitors could be a viable approach in the future to improve management of myopia in a clinical setting.

\section{Supplemental Data}

Supplemental material for this article can be found at https://doi.org/10.1016/j.ajpath.2018.04.011.

\section{References}

1. Grossniklaus HE, Green WR: Pathologic findings in pathologic myopia. Retina 1992, 12:127-133 
2. Chassine T, Villain M, Hamel CP, Daien V: How can we prevent myopia progression? Eur J Ophthalmol 2015, 25:280-285

3. Li X, Wu M, Zhang L, Liu H, Zhang L, He J: Riboflavin and ultraviolet A irradiation for the prevention of progressive myopia in a guinea pig model. Exp Eye Res 2017, 165:1-6

4. Wollensak G, Spoerl E: Collagen crosslinking of human and porcine sclera. J Cataract Refract Surg 2004, 30:689-695

5. Campbell IC, Hannon BG, Read AT, Sherwood JM, Schwaner SA, Ethier CR: Quantification of the efficacy of collagen cross-linking agents to induce stiffening of rat sclera. J R Soc Interface 2017, 14: 20170014

6. Chu Y, Cheng Z, Liu J, Wang Y, Guo H, Han Q: The effects of scleral collagen cross-linking using glyceraldehyde on the progression of form-deprived myopia in guinea pigs. J Ophthalmol 2016, 2016: 3526153

7. Iseli HP, Korber N, Koch C, Karl A, Penk A, Huster D, Reichenbach A, Wiedemann P, Francke M: Scleral cross-linking by riboflavin and blue light application in young rabbits: damage threshold and eye growth inhibition. Graefes Arch Clin Exp Ophthalmol 2016, 254:109-122

8. Norton T, Miller E: Collagen and protein-levels in sclera during normal development, induced myopia, and recovery in tree shrews. Invest Ophthalmol Vis Sci 1995, 46:S760

9. Zorn M, Hernandez M, Norton T, Yang J, Ye H: Collagen geneexpression in the developing tree shrew sclera. Invest Ophthalmol Vis Sci 1992, 33:S1053

10. McBrien NA: Regulation of scleral metabolism in myopia and the role of transforming growth factor-beta. Exp Eye Res 2013, 114: $128-140$

11. McBrien NA, Cornell LM, Gentle A: Structural and ultrastructural changes to the sclera in a mammalian model of high myopia. Invest Ophthalmol Vis Sci 2001, 42:2179-2187

12. McBrien NA, Gentle A: Role of the sclera in the development and pathological complications of myopia. Prog Retin Eye Res 2003, 22: 307-338

13. McBrien NA, Lawlor P, Gentle A: Scleral remodeling during the development of and recovery from axial myopia in the tree shrew. Invest Ophthalmol Vis Sci 2000, 41:3713-3719

14. Norton T, Kang R: Morphology of tree shrew sclera and choroid during normal development induced myopia, and recovery. Invest Ophthalmol Vis Sci 1996, 37:S1490

15. Phillips JR, Khalaj M, McBrien NA: Induced myopia associated with increased scleral creep in chick and tree shrew eyes. Invest Ophthalmol Vis Sci 2000, 41:2028-2034

16. Rada JA, Nickla DL, Troilo D: Decreased proteoglycan synthesis associated with form deprivation myopia in mature primate eyes. Invest Ophthalmol Vis Sci 2000, 41:2050-2058

17. Rada JA, Shelton S, Norton TT: The sclera and myopia. Exp Eye Res 2006, 82:185-200

18. Zou L, Liu R, Zhang X, Chu R, Dai J, Zhou H, Liu H: Upregulation of regulator of G-protein signaling 2 in the sclera of a form deprivation myopic animal model. Mol Vis 2014, 20:977-987

19. Jones BE, Thompson EW, Hodos W, Waldbillig RJ, Chader GJ: Scleral matrix metalloproteinases, serine proteinase activity and hydrational capacity are increased in myopia induced by retinal image degradation. Exp Eye Res 1996, 63:369-381

20. Guggenheim JA, McBrien NA: Form-deprivation myopia induces activation of scleral matrix metalloproteinase-2 in tree shrew. Invest Ophthalmol Vis Sci 1996, 37:1380-1395

21. Siegwart JT, Norton TT: Steady state mRNA levels in tree shrew sclera with form-deprivation myopia and during recovery. Invest Ophthalmol Vis Sci 2001, 42:1153-1159

22. Yang S, Ye J, Long Q: [Expressions of collagen, matrix metalloproteases-2, and tissue inhibitor of matrix metalloproteinase-2 in the posterior sclera of newborn guinea pigs with negative lensdefocused myopia] Chinese. Zhongguo Yi Xue Ke Xue Yuan Xue Bao 2010, 32:55-59
23. Rada JA, Brenza HL: Increased latent gelatinase activity in the sclera of visually deprived chicks. Invest Ophthalmol Vis Sci 1995, 36: $1555-1565$

24. Rada JA, Perry CA, Slover ML, Achen VR: Gelatinase A and TIMP-2 expression in the fibrous sclera of myopic and recovering chick eyes. Invest Ophthalmol Vis Sci 1999, 40:3091-3099

25. Schippert R, Brand C, Schaeffel F, Feldkaemper MP: Changes in scleral MMP-2, TIMP-2 and TGFbeta-2 mRNA expression after imposed myopic and hyperopic defocus in chickens. Exp Eye Res 2006, 82:710-719

26. Bates AL, Pickup MW, Hallett MA, Dozier EA, Thomas S, Fingleton B: Stromal matrix metalloproteinase 2 regulates collagen expression and promotes the outgrowth of experimental metastases. J Pathol 2015, 235:773-783

27. Hori Y, Kashimoto T, Yonezawa T, Sano N, Saitoh R, Igarashi S, Chikazawa S, Kanai K, Hoshi F, Itoh N, Higuchi SI: Matrix metalloproteinase-2 stimulates collagen-I expression through phosphorylation of focal adhesion kinase in rat cardiac fibroblasts. Am J Physiol Cell Physiol 2012, 303:C947-C953

28. Onozuka I, Kakinuma S, Kamiya A, Miyoshi M, Sakamoto N, Kiyohashi K, Watanabe T, Funaoka Y, Ueyama M, Nakagawa M, Koshikawa N, Seiki M, Nakauchi H, Watanabe M: Cholestatic liver fibrosis and toxin-induced fibrosis are exacerbated in matrix metalloproteinase-2 deficient mice. Biochem Biophys Res Commun 2011, 406:134-140

29. Radbill BD, Gupta R, Ramirez MC, DiFeo A, Martignetti JA, Alvarez CE, Friedman SL, Narla G, Vrabie R, Bowles R, Saiman Y, Bansal MB: Loss of matrix metalloproteinase-2 amplifies murine toxin-induced liver fibrosis by upregulating collagen I expression. Dig Dis Sci 2011, 56:406-416

30. Jia Y, Hu DN, Zhu D, Zhang L, Gu P, Fan X, Zhou J: MMP-2, MMP3, TIMP-1, TIMP-2, and TIMP-3 protein levels in human aqueous humor: relationship with axial length. Invest Ophthalmol Vis Sci 2014, 55:3922-3928

31. Zhuang H, Zhang R, Shu Q, Jiang R, Chang Q, Huang X, Jiang C, $\mathrm{Xu}$ G: Changes of TGF-beta2, MMP-2, and TIMP-2 levels in the vitreous of patients with high myopia. Graefes Arch Clin Exp Ophthalmol 2014, 252:1763-1767

32. Qian L, Zhao H, Li X, Yin J, Tang W, Chen P, Wang Q, Zhang J: Pirenzepine inhibits myopia in guinea pig model by regulating the balance of MMP-2 and TIMP-2 expression and increased tyrosine hydroxylase levels. Cell Biochem Biophys 2015, 71:1373-1378

33. Liu HH, Kenning MS, Jobling AI, McBrien NA, Gentle A: Reduced scleral TIMP-2 expression is associated with myopia development: TIMP-2 supplementation stabilizes scleral biomarkers of myopia and limits myopia development. Invest Ophthalmol Vis Sci 2017, 58: 1971-1981

34. Liu HH, Gentle A, Jobling AI, McBrien NA: Inhibition of matrix metalloproteinase activity in the chick sclera and its effect on myopia development. Invest Ophthalmol Vis Sci 2010, 51:2865-2871

35. Itoh $\mathrm{T}$, Ikeda $\mathrm{T}$, Gomi H, Nakao $\mathrm{S}$, Suzuki $\mathrm{T}$, Itohara $\mathrm{S}$ : Unaltered secretion of beta-amyloid precursor protein in gelatinase A (matrix metalloproteinase 2)-deficient mice. J Biol Chem 1997, 272: 22389-22392

36. Schaeffel F, Burkhardt E, Howland HC, Williams RW: Measurement of refractive state and deprivation myopia in two strains of mice. Optom Vis Sci 2004, 81:99-110

37. Zhou X, Ji F, An J, Zhao F, Shi F, Huang F, Li Y, Jiao S, Yan D, Chen X, Chen J, Qu J: Experimental murine myopia induces collagen type Ialphal (COL1A1) DNA methylation and altered COL1A1 messenger RNA expression in sclera. Mol Vis 2012, 18 : $1312-1324$

38. Livak KJ, Schmittgen TD: Analysis of relative gene expression data using real-time quantitative PCR and the 2(-Delta DeltaC(T)) method. Methods 2001, 25:402-408

39. Chen S, Zhi Z, Ruan Q, Liu Q, Li F, Wan F, Reinach PS, Chen J, Qu J, Zhou X: Bright light suppresses form-deprivation myopia development 
with activation of dopamine D1 receptor signaling in the ON pathway in retina. Invest Ophthalmol Vis Sci 2017, 58:2306-2316

40. Deng HW, Tian Y, Zhou XJ, Zhang XM, Meng J: Effect of bilberry extract on development of form-deprivation myopia in the guinea pig. J Ocul Pharmacol Ther 2016, 32:196-202

41. Pardue MT, Stone RA, Iuvone PM: Investigating mechanisms of myopia in mice. Exp Eye Res 2013, 114:96-105

42. Wisard J, Faulkner A, Chrenek MA, Waxweiler T, Waxweiler W, Donmoyer C, Liou GI, Craft CM, Schmid GF, Boatright JH, Pardue MT, Nickerson JM: Exaggerated eye growth in IRBP-deficient mice in early development. Invest Ophthalmol Vis Sci 2011, 52: 5804-5811
43. Zhou X, Huang Q, An J, Lu R, Qin X, Jiang L, Li Y, Wang J, Chen J, Qu J: Genetic deletion of the adenosine A2A receptor confers postnatal development of relative myopia in mice. Invest Ophthalmol Vis Sci 2010, 51:4362-4370

44. Zhou G, Strom RC, Giguere V, Williams RW: Modulation of retinal cell populations and eye size in retinoic acid receptor knockout mice. Mol Vis 2001, 7:253-260

45. Schippert R, Burkhardt E, Feldkaemper M, Schaeffel F: Relative axial myopia in Egr-1 (ZENK) knockout mice. Invest Ophthalmol Vis Sci 2007, 48:11-17

46. Frisch SM, Morisaki JH: Positive and negative transcriptional elements of the human type IV collagenase gene. Mol Cell Biol 1990, 10:6524-6532 